\title{
Ubiquitous Plant Farming based on networks
}

\author{
Soong-hee Lee *, DongIl Kim **, Heechang Chung ***, Aekyung Moon ****, Song Li**** \\ * Inje Univ., Korea, ** Dongeui Univ., Korea, \\ $* * *$ NIA, Korea, **** ETRI, Korea \\ icshlee@inje.ac.kr, dikim@deu.ac.kr, heechang@nia.or.kr, akmoon@etri.re.kr, li.song@etri.re.kr
}

\begin{abstract}
Ubiquitous Plant Farming based on network is a service which is capable of coping with environmental and technical problems with the actualized IT convergence case for agriculture. This paper presents a brief survey on the standardization status including the reference model, service requirements and network capabilities prepared for this purpose.
\end{abstract}

Keywords — Ubiquitous Plant Farming, IT convergence.

\section{INTRODUCTION}

IT convergence with agriculture is expected to bring more efficiency and quality improvement in producing, distributing, consuming of agricultural products with the aid of information processing and autonomous control technologies of the IT area.

However, there exist many difficulties to establish services and systems to actualize the IT convergence service in the agricultural field to cope with various objects such as timevarying weather changes, growth condition of farm products, and continual diseases or technical problems such as battery life, sensor malfunctions at severe conditions. In addition, the gap of viewpoints between the people engaged in farming and the IT engineers may cause more problems for accomplishing this mission.

Therefore, it is required to consider the actualized IT convergence case for agriculture, namely Ubiquitous Plant Farming as a solution to cope with the presented problems[1]. The standardization process related with this issue is in progress in ITU-Y SG13 with the name of Y.ufn (Ubiquitous Plant Farming based on networks)[2].

It looks meaningful to check each point by presenting an overview including the reference model, service requirements and network capabilities[3][4] for this purpose, though its standardization process is just at the initial stage.

This paper presents the reference model, service requirements, and network capabilities considering different environments for Ubiquitous Plant Farming based on networks in Y.ufn.

\section{REFERENCE MODEL FOR UBIQUITOUS PLANT FARMING BASED ON NETWORKS}

Ubiquitous Plant Farming could be supported by future network services, i.e., farm products theft service, farm products traceability service, remote farm management service, farm production regulation service, as shown in the Figure $1[2]$.

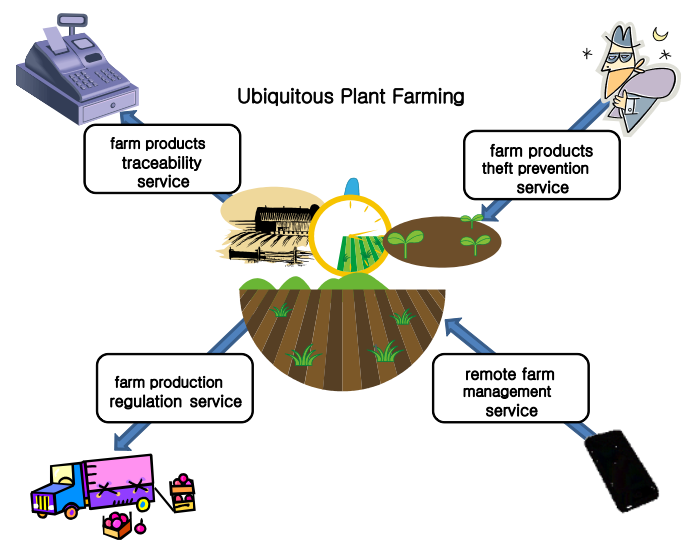

Figure 1. Conceptual diagram of Ubiquitous Plant Farming based on networks.

Ubiquitous Plant Farming can be run autonomously without human intervention when we apply the most advanced technologies such as sensors, computers, or control systems. However, there can be possibilities of unexpected events such as products theft, cost-down due to excess production, etc. Hence, we must confront these difficulties by any means. The network-aided support of the following services could solve such problems on the next generation or future network environment as follows:

- farm products theft prevention service: a service that prevents the theft of agricultural products in farmlands, greenhouses, or warehouses by means of surveillance such as CCTV, infrared sensors, or other sensors which are connected to agricultural producers through networks.

- farm products traceability service: a service that provides the traceability information about farm products in the market to customers, through networks, including identity information about agricultural producers, food safety certification, etc. 
- remote farm management service: a service that provides agricultural producers means to monitor and control farm conditions on the remote side through devices such as smartphones or other types of smart terminals connected to networks.

- farm production regulation service: a service that provides agricultural producers the information for deciding which farm products to sow, when to sow, or when to harvest, which are essential for farm production regulation considering at large.

\section{III.SERVICE REQUIREMENTS FOR UBIQUITOUS PLANT FARMING BASED ON NETWORKS}

\section{A. Requirements for ICT convergence with outdoor farming}

ICT convergence technology about raising outdoors crops can be divided into two stages based on an application concept in Y.ufn as follows:

- Cultivation/harvesting stage: A high functional and pro-environmental support technology is important like a growth management and damage prevention support, a productivity and quality prediction. And also, the standard technology development centralizing harvesting high-quality crops, prevention of damage;

- Processing/distribution stage: An integration control technology of production, processing and distribution is important like stability, pro-environment and freshness authentication and tracking.

And also, the standard technology development related in freshness, stability maintenance and value added improvement is in a process of processing and distribution.

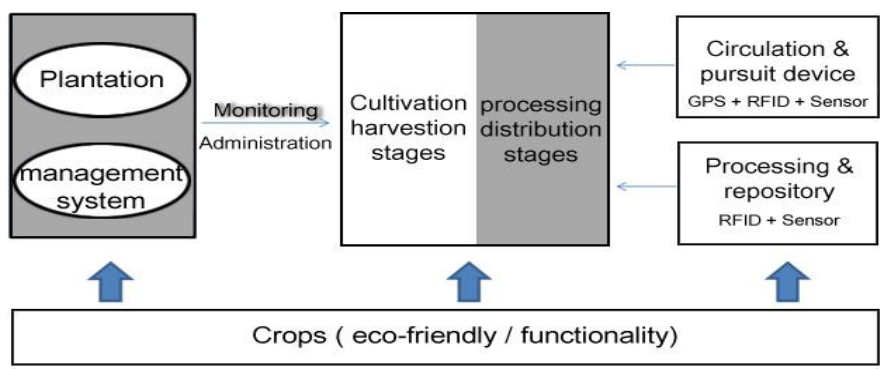

Figure 2. Configuration of stages for ICT convergence with outdoors farming.

\section{B. Requirements for ICT convergence with greenhouse farming}

As an application concept of IT convergence technology about greenhouse farming, the Figure 3 offers technology as follows, through installed sensors and networks:

- Monitoring a change of facility environment in realtime

- Offering the most suitable information of the quality of crops by analyzing synthetically the observation of crops change, growth condition, and environment information.

- Organically control facility environment according to a growth level of crops and a change of environment.

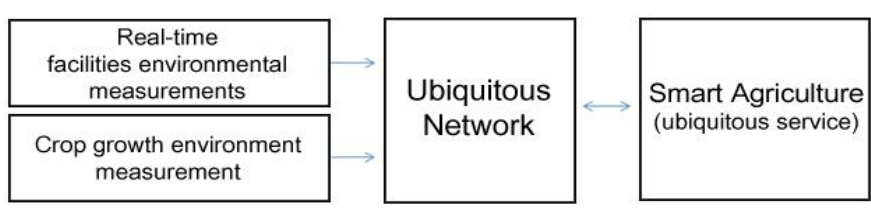

Figure 3. Configuration for ICT convergence with greenhouse farming.

\section{Requirements for ICT convergence with plant factory}

Required features in a plant factory consist of devices related in energy, harvesting device, a light source, environment control, and etc. for a plant factory interior, and a wide area of interfaces between database and a plant factory for related control information can be divided according to ICT fields. Requirement profile for environment control and monitoring system in plant factory are shown in the Figure 4.

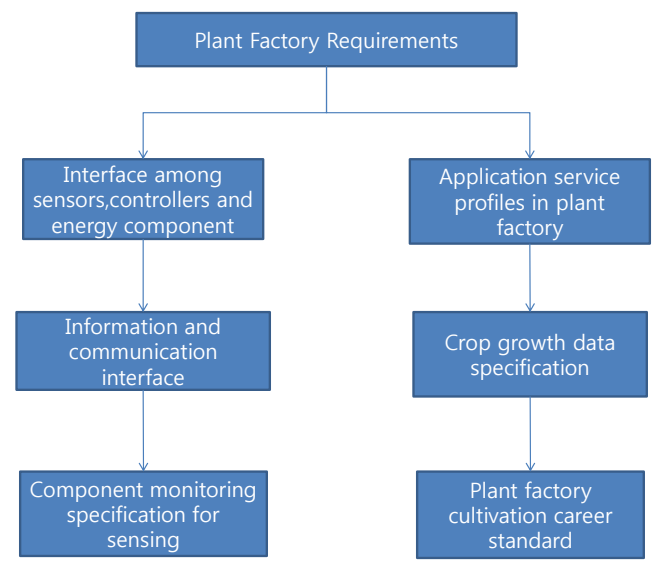

Figure 4. Plant factory requirements according to ICT fields.

\section{IV.NETWORK CAPABILITIES FOR UBIQUITOUS PLANT FARMING BASED ON NETWORKS}

The high-level capabilities for the support of Ubiquitous Plant Farming are listed in Y.ufn as follows

- Connecting to anything capabilities: The capabilities of "connecting to anything" refer to the support of the different ubiquitous networking communication types(person-to-person communication, person-toobject communication, and object-to-object communication) and include the support of tag-based devices (e.g., RFID) and sensor devices. Identification, 
naming, and addressing capabilities are essential for supporting "connecting to anything".

- Open web-based service environment capabilities: Emerging ubiquitous services/applications will be provided based upon an open web-based service environment as well as on legacy telecommunication and broadcasting services. In particular, application programming interface (API) and web with dynamics and interactivities will be supported. Such a web-based service environment will allow not only the creation of retail community-type services but also the building of an open service platform environment which thirdparty application developers can access to launch their own applications. Using interactive, collaborative and customizable features, the web can provide rich user experiences and new business opportunities for the provision of ubiquitous networking services and applications.

- Context-awareness and seamlessness capabilities: Context-aware means the ability to detect changes in the status of objects. Intelligence systems associated with this capability can help to provide the best service which meets the situation using user and environmental status recognition. Seamlessness is a key capability for "5Any" (i.e., anytime, anywhere, any service, any network, and any object).

- Multi-networking capabilities: Transport stratum needs multi-networking capabilities in order to simultaneously support unicast/multicast, multihoming, and multi-path, etc. Because of high traffic volume and the number of receivers, ubiquitous networking requires multicast transport capability for resource efficiency. Multi-homing enables the device to be always best connected using multiple network interfaces including different fixed/mobile access technologies. These capabilities can improve network reliability and guarantee continuous connectivity with desirable QoS through redundancy and fault tolerance.

- End-to-end connectivity over interconnected networks: For ubiquitous plant farming, it is critical to develop the solution to provide end-to-end connectivity to all objects over interconnected heterogeneous networks such as NGN, other IP-based networks, broadcasting networks, mobile/wireless networks, PSTN/ISDN, etc. IPv6, with its large address space, can be considered a good candidate for providing globally unique addresses to objects.

\section{Conclusions}

A survey on the standardized points around Ubiquitous Plant Farming based on networks is given in this paper. The standardization work for Ubiquitous Plant Farming based on networks is just at the beginning stage. Hence, more studies on each point are required to finish the works including amendments and enhancements. More interests are expected to attain the successful results that ultimately contribute to innovate in the lifestyle.

\section{REFERENCES}

[1] Se-Han Kim, et. al., Standardization Trend of_Agriculture-IT Convergence Technology in Korea, IT Convergence and Services Lecture Notes in Electrical Engineering Volume 107, 2011, pp 265-274

[2] TD55-Y.ufnsc, ITU-T SG13, Revised text of the draft Recommendation "Overview of Ubiquitous Plant Farming based on networks, Jun. 2013.

[3] Recommendation ITU-T Y.2012, Functional requirements and architecture of next generation networks, Sept. 2006.

[4] Recommendation ITU-T Y.2201, Requirements and capabilities for ITU-T NGN, Sept. 2009. 